\title{
Cyclopentenone-containing oxidized phospholipids and their isoprostanes as pro-resolving mediators of inflammation
}

\author{
Olivier Friedli and Stefan Freigang \\ Institute of Pathology, University of Bern, CH-3010 Bern, Switzerland
}

Address correspondence to:

\author{
PD Dr. Stefan Freigang \\ Immunopathology IP-2 \\ Institute of Pathology \\ University of Bern \\ Murtenstrasse 31 \\ $\mathrm{CH}-3010$ Bern \\ Switzerland \\ Phone: $\quad+41-31-6323205$ \\ Email: stefan.freigang@pathology.unibe.ch
}

Keywords:

cyclopentenone, inflammation, isoprostane, Nrf2, oxidized phospholipid, resolution

\begin{abstract}
Abbreviations:
15d-PGJ2, 15-deoxy- $\Delta 12,14$-prostaglandin J2; ARE, antioxidant response element; COX, cyclooxygenase; DAMP, damage-associated molecular pattern; DC, dendritic cell; EC, epoxycyclopentenone; EI, epoxyisoprostane; ICAM, intercellular adhesion molecule; IFN- $\gamma$, interferon-gamma; IL, interleukin; iNOS, inducible nitric oxide synthase; KOdiAPC, 1-palmitoyl-2-(5-keto-6-octene-dioyl)-sn-glycero-3-

phosphocholine; Nrf2, nuclear factor E2-related factor 2; OxLDL, oxidized low density lipoprotein; OxPAPC, oxidized PAPC; OxPL, oxidized phospholipid; PAMP, pathogen-associated molecular patter; PAPC, 1-palmitoyl-2-arachidonoyl-sn-glycero3-phosphocholine; PECPC, 1-palmitoyl-2-(5,6-epoxyisoprostane A2)-sn-glycero-3phosphocholine; PEIPC, 1-palmitoyl-2-(5,6-epoxyisoprostane E2)-sn-glycero-3phosphocholine; PG, prostaglandin; PGD2S, prostaglandin D2 synthase; PGPC, 1palmitoyl-2-glutaroyl-sn-glycero-3-phosphocholine; POVPC, 1-palmitoyl-2-(5oxovaleroyl)-sn-glycero-3-phosphocholine; PPAR- $\gamma$, peroxisome proliferator activated receptor-gamma; PRR, pattern recognition receptor; RSV, respiratory syncytial virus; SPM, specialized pro-resolving mediator; Th, T helper; TLR, Toll-like receptor; TNF $\alpha$, tumor necrosis factor alpha; VCAM, vascular cell adhesion molecule.
\end{abstract}




\section{Abstract.}

Inflammation represents a powerful innate immune response that defends tissue homeostasis. However, the appropriate termination of inflammatory processes is essential to prevent the development of chronic inflammatory disorders. The resolution of inflammation is actively induced by specialized pro-resolving lipid mediators, which include eicosanoids, resolvins, protectins and maresins. The responsible pro-resolution pathways have emerged as promising targets for anti-inflammatory therapies since they mitigate excessive inflammation without compromising the anti-microbial defenses of the host. We have recently shown that the lipid peroxidation of membrane phospholipids, which is associated with inflammatory conditions, generates oxidized phospholipid (OxPL) species with potent pro-resolving activities. These pro-resolving OxPLs contain a cyclopentenone as their common determinant, and are structurally and functionally related to endogenous proresolving prostaglandins. Here, we review the regulation of inflammatory responses by OxPLs with particular focus on the bioactivities and structural characteristics of cyclopentenone-OxPLs, and discuss the impact of the responsible signaling pathways on inflammatory diseases. 


\section{Introduction}

In order to survive, our organism has to maintain and defend a constant internal milieu while being exposed to continuously changing environmental influences. Inflammation represents a physiological response of the innate immune system to perturbations of this internal balance, irrespective of whether they originate from microbial infection, traumatic injury or metabolic dysfunction [1]. The inflammatory response aims to eliminate or sequester the injuring agent, to mount appropriate wound healing and repair programs and to restore tissue homeostasis. Inflammatory responses are initiated by innate sensing mechanisms that detect barrier breach, loss of cellular integrity or the presence of microbial infection. For this purpose, tissue-resident sentinel cells of the immune system express a panel of specialized pattern recognition receptors (PRRs). These receptors respond to pathogenassociated molecular patterns (PAMPs) that are shared between different microbes, yet are usually not expressed by mammalian cells [2]. In addition, PRRs may also recognize damage-associated molecular patterns (DAMPs), e.g. self-molecules that are produced or released only by stressed or dying cells to alarm the immune system [3]. Activation of PRRs triggers the secretion of diverse inflammatory mediators, including vasoactive substances, pro-inflammatory cytokines, chemokines and phospholipid-derived lipid mediators, which orchestrate the resulting innate immune response. Provided that the immune response succeeds to eliminate the infectious agent or to repair the initial tissue injury, the inflammatory process will be terminated and thus affects tissue function only transiently. However, in cases where the inflammation fails to resolve, for example due to the persistence of a pathogen or the failure to repair the initiating injury or tissue dysfunction [4], the powerful effector functions of the inflammatory response may inflict severe damage on the host tissue. As a result, a sustained inflammatory process develops which can permanently compromise tissue function. Accordingly, non-resolved inflammation has been identified as an important etiologic factor in the pathogenesis of many chronic diseases, including obesity, atherosclerosis, inflammatory bowel disease, neurodegenerative disorders and certain cancers [4]. Therefore, not only the induction of the inflammatory process requires appropriate regulation; also its timely resolution has to be guaranteed in order to avoid the detrimental consequences of chronic inflammation. It is now evident that the resolution of inflammation represents a well-controlled process already programmed during the initiation phase of the inflammatory response and the underlying molecular pathways are currently being elucidated at the molecular level [5]. 


\section{The resolution of inflammation}

The termination of inflammatory responses has been long regarded a passive event that automatically occurs with the removal of the injuring agent and the resulting cessation of pro-inflammatory signals. However, the resolution of inflammation is now recognized as an actively induced process, which is directed by a family of specialized pro-resolving lipid mediators (SPMs) [5,6]. Analogous to the inflammation-inducing prostaglandins and leukotrienes, these pro-resolving mediators are enzymatically generated from phospholipid-derived polyunsaturated fatty acids. They include eicosanoids, such as the lipoxins, but also the more recently discovered resolvins, protectins and maresins that are synthesized from essential omega-3 fatty acids, including eicosapentaenoic acid and docosahexaenoic acid $[5,6]$. Physiological inflammation comprises a sequence of events that begins with the initial release of pro-inflammatory mediators, followed by the subsequent endothelial activation, edema formation and infiltration of polymorphonuclear neutrophils into the injured tissue. The nonphlogistic recruitment of monocytes then supports the clearance of apoptotic neutrophils via efferocytosis and initiates wound repair programs to facilitate the return to tissue homeostasis. Synthesis of proresolving lipid mediators is mediated by cell-cell interactions within the inflammatory exudates. Intriguingly, the generation of these pro-resolving signals appears to be already instructed early during the inflammatory process. This prepares the lipid mediator class switch from the predominant production of pro-inflammatory mediators during the initiation phase towards the production of a pro-resolving mediator profile at later stages, which heralds the resolution of inflammation [6]. Importantly, while SPMs specifically influence different levels of the inflammatory process to promote its resolution, their activity does not compromise host resistance to infection [5]. On the contrary, SPMs have been shown to increase host survival in models of bacterial [7-9], fungal [10] and viral [11] infections. This is a clear difference to conventional anti-inflammatory therapies, which usually also mediate certain immunosuppressive effects. In this regard the pro-resolution mediators are considered particularly advantageous since they could provide novel therapeutic approaches for inflammatory diseases that do not interfere with anti-infectious immune defenses $[12,13]$. Accordingly, the discovery of these resolution-inducing pathways has sparked great interest in identifying the involved lipid species and the molecular mechanisms mediating their signaling. Recent studies suggest that not only the lipid mediators generated by enzymatic reactions, but also certain oxidized phospholipid (OxPL) species that are formed by radical-mediated lipid peroxidation in 
the context of inflammation exert potent pro-resolving activities in vitro and in vivo. Here, we will summarize the current knowledge of the regulation of inflammatory responses by OxPLs with a particular focus on cyclopentenone-containing OxPLs, which have emerged as powerful pro-resolving lipid mediators with high therapeutic potential.

\section{Oxidative stress and the generation of oxidized phospholipids}

The generation of reactive oxygen species (ROS) represents an essential innate effector function of phagocytic cells that is required for the successful immune defense against microbial pathogens. In non-phagocytic cells, ROS serve as important intracellular signaling molecules that modulate the activity of biological processes. For instance, transient increases in cellular ROS levels are involved in the regulation of cellular proliferation, differentiation, migration and angiogenesis responses, as well as the induction of cell death programs [14]. Likewise, the intracellular redox balance also influences the activity of signaling pathways via the reversible modification of ROS-sensitive cysteine residues [14]. Nevertheless, conditions in which the generation of ROS exceeds the protective capacity of the cellular antioxidant defense mechanisms, as may occur during inflammation, are associated with oxidative stress. The excessive ROS production and an imbalanced redox homeostasis are especially harmful due to the ensuing oxidative modification of self-molecules, such as lipids, proteins or nucleic acids. Accordingly, oxidative stress has been identified as an important etiologic factor in the pathogenesis of chronic inflammatory diseases, metabolic diseases and cancer $[15,16]$.

The exposure of biological membranes to ROS rapidly leads to the oxidative modification of membrane phospholipids (Fig.1). Particularly the polyunsaturated fatty acid side chains of phospholipids are highly susceptible to radical-mediated oxidation. Importantly, this lipid peroxidation is non-specific, and thus generates a complex mixture of distinct oxidized phospholipid species with diverse bioactivities [17]. The radical-mediated oxidation of membrane phospholipids can produce two major categories of OxPLs (Fig.2) [17,18]. OxPLs of the first category are characterized by a truncated oxidized fatty acid at the sn2 position that contains hydroxyl or carbonyl groups. These OxPLs are generated due to a fragmentation of the oxidized fatty acid, which also releases highly reactive short-chain aldehydes, such as 4-hydroxynonenal or malondialdehyde. OxPLs belonging to the second category are a result of intra-molecular cyclization, rearrangements and further 
oxidation; they may contain isoprostanes, isothromboxanes or isofurans at their sn2 position $[17,18]$. Examples for each category of OxPLs have been detected in vivo and characterized as synthetic compounds. While common effects shared by several OxPLs exist, these studies also indicated distinct biological activities for structurally diverse OXPLs. OXPLs have been demonstrated in a range of disease settings with a prominent inflammatory component. Importantly, the pathophysiological relevance of OxPLs to such disease conditions, including acute and chronic infections [19-21], atherosclerosis [22] and neurodegenerative disorders [23] appears to be directly related to their ability to modulate the underlying inflammatory processes (Fig.1).

\section{Pro- and anti-inflammatory activities of oxidized phospholipids}

Although both pro- and anti-inflammatory bioactivities of OxPLs have been described, OxPLs are generally considered to exert strong pro-inflammatory effects and to promote pathogenic inflammation in a range of disease settings [17]. Indeed, many studies have reported such pro-inflammatory activities of OxPLs, which can be attributed to the direct recognition of OxPLs by different PRRs of the innate immune system. In particular, OxPLs have been shown to interact with Toll-like receptors [21,24,25], scavenger receptors [26-28], complement components [23], C-reactive protein [29] and natural antibodies [30,31]. For example, in the acute respiratory distress syndrome caused by acid-induced lung injury or infection with highly pathogenic influenza viruses, locally generated OxPLs initiate the pathogenic cytokine storm by activating TLR4 signaling [21]. In the context of atherosclerosis, vascular inflammation is driven by OxPL species present on oxidized low-density lipoprotein $(O x L D L)$. These OxPLs have been shown to provoke the secretion of proinflammatory cytokines and chemokines by activating a hetero-dimeric complex of TLR4 and TLR6 on macrophages [24]. Moreover, this process required the cooperation of the dimeric TLR4/6 with scavenger receptor CD36, which in addition supported the internalization of OxLDL for the subsequent priming of IL-1beta secretion [32]. A similar co-operation in the recognition of OxPLs has been reported for CD36 and TLR2. This interaction was shown to induce the expression of proinflammatory mediators in macrophages [33]. Furthermore, it was found to contribute to apoptosis induction by OxPLs in foam cells [25]. Besides the direct PRR recognition of OxPLs, immune activation in response to phospholipid oxidation can also result from the PRR recognition of so-called "oxidation-specific epitopes", which are formed during the oxidative modification of phospholipids. Oxidation-specific 
epitopes include the phosphocholine moiety revealed on oxidized PC but absent from native PC, as well as different types of OxPL-protein adducts that are generated during lipid peroxidation [34]. Intriguingly, these epitopes are shared between OxPLs, apoptotic cells, damaged proteins and also microbial cell walls [35]. Their recognition by diverse PRRs has been implicated in the silent removal of cellular debris, yet may also contribute to anti-microbial immune responses [34].

Contrasting the strong pro-inflammatory responses evoked by OxPLs upon direct recognition by PRRs, a series of reports demonstrated that OxPLs mediate potent anti-inflammatory effects by interfering with the activation of TLRs in response to microbial ligands. For instance, treatment of endothelial cells with OxPLs has been shown to interfere with the expression of vascular adhesion molecules and the neutrophil adhesion induced by LPS [36]. A similar inhibition of TLR4 signaling was observed in vivo and protected mice from lethal endotoxin shock [19]. In this study, the anti-inflammatory activity was attributed to the ability of OxPL to block the interaction of LPS with LBP and CD14 [19]. Still, the immunomodulatory effects of OxPL are not limited to particular TLRs. Several studies have demonstrated the ability of OxPL to inhibit the signaling of various TLRs in myeloid cells [37-40]. In addition, OxPL were found to negatively regulate dendritic cell maturation and reduce the T cell stimulatory capacity of dendritic cells [38]. An in vivo correlate for these observations has been reported for mycobacterial infection, where locally generated OxPLs were found to inhibit the presentation of bacterial antigens to $T$ lymphocytes via the MHC and CD1d presentation pathways [20]. Thus, OxPLs have been demonstrated to trigger vigorous inflammation by activating innate PRRs, but paradoxically may also dampen inflammatory responses by interfering with TLR signaling.

\section{Immunomodulatory bioactivities of individual oxidized phospholipid species}

The complexity of bulk OxPL preparations and the diversity of the immune phenotypes observed with such OxPL mixtures highlight the importance of studying the biological effects of OxPLs using defined synthetic OxPL species. Originally, OxPLs were characterized as the bioactive components of OxLDL that are responsible for many of its pro-atherogenic effects [22]. As such, OxPL were shown to stimulate endothelial cell-monocyte interactions [41-43] and induce the secretion of the pro-inflammatory chemokines IL-8 and MCP-1 in endothelial cells [44-46]. 
However, experiments performed with the individual purified or synthetic OxPL species revealed that these bioactivities were not specific for particular OxPLs, but rather appeared to be shared by structurally diverse OxPLs [41-46]. At the same time, these OxPLs also exhibited anti-inflammatory activities since they inhibited TLR4-induced E-selectin expression on endothelial cells and protected mice from LPS-induced shock in vivo [19,36]. A potential explanation for the ability of structurally diverse OxPLs to elicit both pro- and anti-inflammatory effects in endothelial cells was offered by a recent study [47]. Oskolkova et al. demonstrated that the anti-inflammatory effects of OxPLs are already induced at the low concentrations of OxPLs that may be present in the circulation in vivo. However, substantially higher OxPL concentrations were required to elicit the pro-inflammatory cytokine responses from endothelial cells. This suggested that the pro-inflammatory bioactivity might be restricted to localized processes, such as the atheroma, in which OxPLs may be much more abundant $[17,47]$. Nevertheless, in vitro studies investigating the effects of OxPLs on endothelial barrier function revealed specific effects for structurally different OxPLs [48].

The bioactivities of individual OxPLs appear, however, to be more specific in myeloid cells. The truncated OxPLs KOdiAPC and POVPC were shown to promote the apoptosis of macrophage-foam cells by activating TLR2 and CD36 (Fig.2) [25]. Still, neither of the truncated OxPLs POVPC and PGPC contributed to the OxPL-induced expression of pro-inflammatory genes via TLR2 [33]. Both POVPC and PGPC were found to inhibit phagocytosis and bacterial clearance in vivo [49]. On the contrary, the cyclized OxPLs PEIPC and PECPC but not the truncated OxPLs POVPC, PGPC or KOdiAPC inhibited the pro-inflammatory cytokine responses in TLR-activated dendritic cells [40]. Similarly, PEIPC was shown to inhibit secretion of TNF $\alpha$ and to modulate the TLR2-induced IL-12 and IL-10 responses in mycobacterial infection in vivo [20]. In addition, PEICP but not POVPC or PGPC interfere with the presentation of mycobacterial antigens to T cells [20] and influences the capacity of DCs to support differentiation of CD4+ T cells towards the pro-inflammatory Th1 subset [40]. Together, these findings indicate that structurally distinct OxPL species exhibit very defined and non-overlapping bioactivities. Although some results suggest that effects of OxPLs on endothelial cells may be related rather to local lipid concentrations than to specific structural characteristics; other experiments have clearly shown distinct effects of structurally diverse OxPLs on cytokine responses, cellular activation and differentiation, antigen presentation and phagocytosis. 


\section{Cyclopentenone-OxPLs are powerful pro-resolving mediators}

Recent studies have shown that the anti-inflammatory bioactivity of bulk OxPL mixtures is primarily mediated by OxPL species containing a cyclopentenone moiety as their defining structural component (Fig.2) [40]. By examining the bioactivity and composition of in vitro generated bulk OxPL preparations, we identified PECPC and PEIPC as the most active mediators with anti-inflammatory bioactivity. Results suggested that PEIPC likely represents a precursor to the more active PECPC $[42,50,51]$. The cyclopentenone-OxPL PECPC exhibited strong pro-resolving bioactivity in vitro and in vivo. In particular, exposure to PECPC effectively suppressed the TLR-induced production of inflammatory cytokines and chemokines in dendritic cells. Furthermore, PECPC strongly reduced endothelial cell-leukocyte interactions and abrogated pulmonary inflammation in an in vivo model of sepsisassociated lung injury [40]. PECPC was first identified by Watson et al. as a bioactive component of minimally modified LDL [42] and later studies demonstrated the generation of biologically relevant levels od PECPC in IL-1 $\beta$-stimulated human aortic endothelial cells in vitro [46]. Remarkably, the sn2-oxidized fatty acid present in PECPC, which is a 5,6-epoxyisoprostane A2 - termed epoxycyclopentenone (EC) here, exhibits close structural homology to the endogenous pro-resolving lipid mediator, 15-deoxy- 1 12,14-prostaglandin J2 (15d-PGJ2). In fact, EC is very likely the bioactive component of PECPC that can be generated by non-enzymatic deacylation of the OxPL in vivo [52].

EC and 15d-PGJ2 share the cyclopentenone moiety, an $\alpha, \beta$-unsaturated carbonyl group that can form covalent adducts with cysteine-residues of cellular proteins via Michael addition. Thereby, cyclopentenone-isoprostanes and cyclopentenoneprostaglandins are capable of activating important redox sensitive signaling pathways, including the antioxidant response. The transcriptional master regulator of the antioxidant response, Nrf2, is kept in the cytosol in an inactive form by its chaperone Keap-1 and is constantly targeted for proteasomal degradation (Fig.3). Upon modification of redox-sensitive cysteine residues of Keap-1 by electrophiles, such as EC or 15d-PGJ2, Nrf2 is released and can initiate the transcription of genes containing an antioxidant response element (ARE) in their promoter region [53]. Analysis of Nrf2-signaling-deficient EC variants as well as of Nrf2-deficient mice demonstrated that the potent pro-resolving bioactivity of cyclopentenone-OxPL and their respective isoprostanes is essentially mediated via the Keap1/Nrf2-signaling 
pathway (Fig.3) [40,54]. Notably, the pro-resolving mediators lipoxin B4 and resolvin D2, that lack the cyclopentenone moiety, neither activated Nrf2-signaling nor induced comparable anti-inflammatory effects in dendritic cells as the cyclopentenone-OxPLs [40]. Nevertheless, electrophilic oxygenated metabolites of docosahexaenoic acid have been reported to mediate certain pro-resolving effects via Nrf2 [55,56], indicating that Nrf2-signaling might represent a common pro-resolution pathway that cyclopentenones share with other electrophilic SPMs derived from docosahexaenoic acid.

\section{Structure-activity-relationship studies of cyclopentenone-isoprostanes}

The radical-mediated oxidation of phospholipids generates cyclized OxPLs containing cyclopentenone-isoprostanes with high structural and functional similarity to the endogenous prostaglandin 15d-PGJ2 (Fig.4). Intriguingly, biological investigations revealed that such OxPL-derived cyclopentenone-isoprostanes exhibit much higher pro-resolving potency than 15d-PGJ2 in vitro and in vivo [40]. A series of studies has recently probed the structure-activity-relationship of cyclopentenoneisoprostanes utilizing a library of novel synthetic variants of the OxPL-derived cyclopentenone-isoprostane EC and of endogenous 15d-PGJ2 [40,51,54]. Their results established the critical importance of the endocyclic enone as the key structural feature that imparts the potent pro-resolving effects of cyclopentenone-isoprostanes $[40,54]$. Furthermore, these experiments also identified important additional structural determinants that modulate the overall biologic activity of these cyclopentenonelipids, thus providing a rationale for the superior efficacy of OxPL-derived cyclopentenone-isoprostanes as compared to $15 d-P G J 2$, and potentially explaining the much weaker pro-resolving activity of other cyclopentenone-prostaglandins, such as PGA2 or PGJ2 (Fig.2). In particular, the bioactivity of EC is mediated by its strong electrophilic nature, which confers the ability to undergo conjugate addition reactions with nucleophiles, such as the thiolate cysteine residues of Keap1. Yet, EC contains several electrophilic sites that might contribute to this reactivity: an endocyclic enone, an exocyclic enone as well as an allylic epoxide (Fig.4). Analysis of synthetic variants lacking individual electrophilic sites demonstrated the importance of the endocyclic enone. For example, removal of the endocyclic enone both abrogated the ability of the respective variant to activate Nrf2 and abolished its pro-resolving potential in vivo. Moreover, introduction of another electrophilic group at this position restored its bioactivity $[40,51]$. These observations were further corroborated using the 
corresponding synthetic variants of 15d-PGJ2 [54], thus confirming the endocyclic enone as the main driver of the pro-resolving bioactivity of cyclopentenones. In comparison, removal of the exocyclic enone from EC or 15d-PGJ2 affected the bioactivity of respective variants to a lesser degree, suggesting that presence of the cross-conjugated enone in EC and 15d-PGJ2 mainly enhances the electrophilic properties of the endocyclic enone [40,54]. Likewise, the absence of this crossconjugated enone might explain the weaker anti-inflammatory activity of the cyclopentenone-prostaglandins PGA1, PGA2 and PGJ2 [58,59]. Conversely, introduction of a cross-conjugated enone into PGA1 and PGJ2 was shown to increase the biological activity of the resulting $\triangle 7-P G A 1$ and $\triangle 12-P G J 2$ variants[60,61].

An important modifying factor determining the enhanced potency of EC versus $15 d-$ PGJ2 appears to be the location of the carboxylic acid on the $\alpha$-side chain and the additional presence of the allylic epoxide in EC [40,54]. Removal of the epoxide or transposition of the carboxylic acid to the $\omega$-side chain reduced the overall bioactivity of the resulting variants [51]. These observations suggested that the ability of EC to undergo an intramolecular reaction between the epoxide and the carboxylic acid which potentially forms the 6-membered lactone present in cyclo-EC - is central to its greatly enhanced bioactivity (Fig.4) [51]. In comparison, generation of such an intramolecular lactone would not be possible in case of 15d-PGJ2 (Fig.4). Indeed, the synthetic EC analogue cyclo-EC containing this lactone exhibited a greatly increased pro-resolving potency in vitro and in vivo [40], which suggested that the ability of OxPL-derived cyclopentenone-isoprostanes to form a stable lactone may explain their enhanced potency. Notably, the non-enzymatic deacylation of OxPL has been shown to occur spontaneously under physiological conditions [52]. This process was found to generate similar intramolecular lactones [62]. Taken together, these findings characterize structural determinants that explain the superior potency of cyclopentenone-isoprostanes as compared to endogenous cyclopentenone-prostaglandins. Furthermore, they establish the OxPL-derived cyclopentenoneisoprostanes, such as EC and cyclo-EC, as promising leads for the development of powerful pro-resolving therapeutics in the future. 


\section{The pro-resolving signaling of cyclopentenones}

The signaling and in vivo bioactivities of cyclopentenone-containing lipid mediators have been best studied for the endogenous cyclopentenone 15d-PGJ2 [63-65]. Initially, the ability of cyclopentenone-prostaglandins inhibit pro-inflammatory macrophage responses, such as pro-inflammatory cytokine production [66] or iNOS expression [67], was proposed to be mediated via nuclear receptor PPAR- $\gamma$, which also transduces their effects on adipose differentiation and lipid metabolism $[68,69]$. However, later studies demonstrated that the anti-inflammatory effects of 15d-PGJ2 were independent of PPAR- $\gamma$ [70]. Instead, the electrophilic cyclopentenoneprostaglandins function as strong pro-resolving mediators by modifying critical cysteine residues of cellular signaling molecules. In particular, 15d-PGJ2 has been shown to inactivate IKK $\beta$, thereby blocking NFKB-signaling[58]. Furthermore, 15dPGJ2 blocks NFKB-dependent transcription in activated macrophages by modifying the DNA binding domains of NFאB subunits [71,72]. In addition, 15d-PGJ2 was shown to mediate powerful pro-resolving effects by covalently binding to specific cysteine residues of Keap1, thus triggering Nrf2-dependent gene expression [73,74].

The generation of different prostaglandins during innate responses is controlled by the coordinated activity of COX-2 and specific prostaglandin synthases, including PGD2 synthase (PGD2S), which balances their pro- and anti-inflammatory activities. Gilroy et al. demonstrated the biphasic expression of COX-2 during self-resolving inflammation. In the rat model of pleurisy, an early peak of COX-2 activity was associated with a predominant generation of PGE2 during the initiation phase and primarily exerted pro-inflammatory effects. In contrast, COX-2 expression rather supported the production of $15 \mathrm{~d}-\mathrm{PGJ} 2$ in the late stages and thereby promoted the termination of the inflammatory process [75]. Hematopoietic PGD2S is expressed in professional antigen presenting cells, including dendritic cells and several types of tissue macrophages, which suggests that these cells can modulate inflammatory responses by producing PGD2 for the subsequent generation of 15d-PGJ2 [76]. In addition, endothelial cells express lipocalin-type PGD2S and produce PGD2 and 15d-PGJ2 upon exposure to laminar shear stress [77]. A study by Rajakariar et al. highlighted the importance of PGD2S-produced PGD2 and 15d-PGJ2 for the physiological initiation and resolution of inflammation, respectively. In a model of zymosan-induced peritonitis, removal of hPGD2S completely abrogated PGD2 and 15d-PGJ2 production in inflammatory exudates [78]. As a consequence, hPGD2S- 
deficient mice initially exhibited larger neutrophilic infiltrates and produced greater amounts of the inflammatory mediators TNF $\alpha$ and MCP-1. Importantly, the lack of 15d-PGJ2 also resulted in an impaired resolution, as hPGD2S-deficient mice displayed an increased accumulation of macrophages and lymphocytes in their peritoneal cavities at later stages [78]. Collectively, these studies emphasize the contribution of endogenously generated cyclopentones to the physiological termination and resolution of inflammation.

Cyclopentenone-prostaglandins have been shown to affect different aspects of resolution, including the modulation of endothelial activation, the inhibition of proinflammatory cytokine and chemokine responses and the resolution of neutrophilic and monocytic infiltrates. The exposure of human umbilical vein endothelial cells to 15d-PGJ2 inhibited the OxLDL-induced expression of the adhesion molecules ICAM1 and E-selectin [79] and reduced the NFKB-activation in response to TNF $\alpha$ [80]. Furthermore, 15d-PGJ2 was shown to inhibit the expression of adhesion molecules and the resulting endothelial cell-monocyte interactions in mouse brain endothelial cells by antagonizing the TNF $\alpha$-induced NFKB signaling [81]. Remarkably, these proresolving effects of $15 d-P G J 2$ were induced independently of PPAR- $\gamma$ [79-81], but could potentially be attributable to the activation of Nrf2 by $15 d-P G J 2$. For example, laminar shear stress enhances the endothelial expression of PGD2S [77] and supports the COX-2/PGD2S-mediated synthesis of PGD2, the precursor of 15dPGJ2 [82]. In parallel, laminar flow has been shown to induce the ARE-mediated expression of signature Nrf2 target genes in human aortic endothelial cells; and the overexpression of Nrf2 or of its target NQO1 reduced the TNF $\alpha$-induced VCAM-1 expression [83]. Supporting this notion, Nrf2 was found to negatively regulate the expression of VCAM-1 in murine arteries at sites exposed to high laminar shear stress in vivo [84]. In human aortic endothelial cells 15d-PGJ2 was shown to form covalent adducts with specific cysteine residues of Keap-1, resulting in the downstream activation of Nrf2-mediated gene expression [82]. Moreover, exogenously applied electrophilic OxPL were demonstrated to trigger the expression of Nrf2 target genes in murine carotid arteries in vivo [85].

In models of acute inflammation, 15d-PGJ2 signaling exerts potent anti-inflammatory effects. For example, 15d-PGJ2 limited the peritoneal mononuclear cell infiltration and inflammation-induced multi-organ damage in a zymosan-induced model of nonseptic shock [86]. Likewise, 15d-PGJ2 was shown to mitigate excessive injuryinduced macrophage activation and pro-inflammatory cytokine responses, which 
protected mice against a subsequent infectious challenge [87]. The studies of ttoh et al. and Mochizuki et al. demonstrated in murine models of pleurisy and acute lung injury that the pro-resolving activity of $15 d-P G J 2$ is mediated via Nrf2 [88,89]. In these models, the induction of inflammation was paralleled by the production of $15 \mathrm{~d}$ PGJ2 in macrophages, which triggered Nrf2-dependent gene expression.

Importantly, both the removal of COX-2-derived endogenous 15d-PGJ2 and genetic ablation of Nrf2-signaling greatly increased the inflammatory infiltrates and impaired the resolution of inflammation, thus indicating that pro-resolving 15d-PGJ2 signaling depends on Nrf2. Accordingly, physiological resolution of inflammation could be restored by exogenous 15d-PGJ2 only in wild type, but not in Nrf2-deficient mice $[88,89]$. Furthermore, cyclopentenone-prostaglandins can also enhance the clearance of inflammatory infiltrates by promoting the apoptosis of leukocytes. For example, pharmacological suppression of $15 \mathrm{~d}-\mathrm{PG}$ formation by inhibition of COX-2 impaired the resolution in pleurisy [90]. This effect was mediated by the defective apoptosis of neutrophils and macrophages in absence of COX-2 activity and could be rescued by the administration of 15d-PGJ2 [90]. The lack of endogenous 15dPGJ2 in PGD2S-deficient mice has been shown to compromise inflammatory resolution and was associated with an enhanced accumulation of F4/80+ macrophages in inflammatory exudates [78]. In vitro studies indicated that 15d-PGJ2 promotes the apoptosis of activated RAW cell macrophages by enhancing the p38 mitogenactivated protein kinase-dependent superoxide production [91]. Another study demonstrated the ability of $15 \mathrm{dPGJ} 2$ to induce apoptosis in human neutrophils via a caspase-dependent but PPAR- $\gamma$-independent mechanism [92]. Still, 15d-PGJ2 signaling does not only act on innate immune cells in the resolving exudates, but may also influence the pro-inflammatory capacity of adaptive T cells. In a model of delayed type hypersensitivity, endogenous 15d-PGJ2 was shown to control the severity and duration of inflammation by limiting the proliferation and cytokine production of pro-inflammatory Th1 lymphocytes [93]. Additionally, 15d-PGJ2 and OxPL-derived cyclopentenone-isoprostanes were found to limit the in vitro differentiation of pro-inflammatory CD4+ Th1 cells by triggering Nrf2 in professional antigen presenting cells [40].

\section{Pro-resolving effects of Nrf2-signaling in inflammation}

The essential role of Nrf2 in mediating the pro-resolving effects of cyclopentenoneOxPLs and 15d-PGJ2 implies that cyclopentenone-OxPLs may also regulate 
inflammatory processes in other diseases influenced by Nrf2-signaling for which the contribution of OxPLs has not yet been recognized. Specifically the aggravated inflammatory phenotype that has been observed in Nrf2-deficient mice in a range of experimental models - including acute tissue injury, microbial infections and chronic metabolic inflammation - strongly suggests that Nrf2-signaling controls the inflammatory tone in response to endogenously generated cyclopentenoneisoprostanes or cyclopentenone-prostaglandins. For example, in the setting of endotoxin-induced or cecal ligation and puncture-induced septic shock, Nrf2signaling was shown to prevent excessive inflammation and severe immunopathology. In contrast, Nrf2-deficient animals exhibited an increased pulmonary leukocyte infiltration, exacerbated production of pro-inflammatory cytokines and increased mortality [94]. Similarly, the absence of Nrf2 also compromised the resolution of inflammation in carageenin-induced pleurisy and acute lung injury, resulting in the impaired clearance of neutrophils, a delayed recruitment of macrophages and enhanced tissue injury $[88,89]$. Notably, the dysfunctional resolution in Nrf2-deficient mice was related to their inability to respond to endogenously generated cyclopentenone-prostaglandins $[88,89]$. The analysis of Nrf2 function specifically in myeloid cells further emphasized its critical role for preventing exaggerated immune responses, organ injury and overall mortality in severe sepsis [95]. In these experiments, Nrf2 was constitutively activated in macrophages and neutrophils via the genetic ablation of Keap-1, which effectively limited systemic inflammation and immunopathology and significantly improved survival. Conversely, the conditional inactivation of Nrf2 in myeloid cells increased the production of pro-inflammatory IL-6, TNF $\alpha$ and MCP-1, aggravated the tissue damage and enhanced disease-associated mortality [95].

In a similar manner, Nrf2 essentially contributes to the resolution of inflammation during microbial infections by limiting excessive immune activation and controlling the extent of tissue damage. In Staphylococcus aureus pneumonia, Nrf2-deficient animals showed larger inflammatory infiltrates and extensive lung injury in combination with an exacerbated production of IL-1 $\beta$, MCP-1 and TNF $\alpha$ [96]. The lack of Nrf2-signaling was found to suppress the transcriptional regulation of mitochondrial biogenesis required for the resolution of lung injury. In a model of chronic lung inflammation triggered by Haemophilus influenzae, Nrf2-deficient mice exhibited increased pro-inflammatory cytokine responses and enhanced airway lymphocytic inflammation [97]. In addition, the genetic or pharmacological activation 
of Nrf2 signaling alleviated the chronic inflammation developing in the human $\beta$ globin model of sickle cell disease, which is associated with an enhanced oxidative microenvironment and the increased generation of intravascular ROS [98]. Human monocyte-derived dendritic cells infected with Dengue virus show a rapid induction of NOX-dependent ROS formation. The subsequent Nrf2 activation and resulting expression of antioxidant response genes regulated the ROS levels in infected cells and thereby beneficially modulated the immune and apoptotic responses against viral infection [99]. Also the infection with respiratory syncytial virus (RSV) is associated with substantially increased ROS production that leads to oxidative stress and lung injury. Analysis of RSV-infected Nrf2-deficient mice indicated that Nrf2-dependent responses significantly restricted lipid oxidation, bronchopulmonary inflammation and epithelial damage, yet also enhanced viral clearance [100]. Still, besides triggering Nrf2 as a result of the increased generation of ROS (and potentially of Nrf2-activating OxPL species) that is associated with viral infection, some viruses may directly target Nrf2 in infected cells to promote conditions that are favorable for viral replication. For example, Marburg virus has been shown to induce Nrf2-dependent gene expression by inactivating Keap-1 via its VP24 protein [101]. In contrast, RSV infection was reported to down-regulate the Nrf2-dependent transcription of antioxidant response genes by promoting the deacetylation and increased proteasomal degradation of Nrf2 [102]. Taken together, these findings suggest that the pro-oxidant environment in acute tissue injury or during microbial infection triggers Nrf2-dependent pro-resolution responses, which in turn restrain oxidative injury, excessive immune activation and resulting immunopathology. Whether and to which extent Nrf2-activation under these conditions involves the generation and activity of cyclopentenone-isoprostanes remains to be investigated.

While the activation of Nrf2-dependent gene expression confers mainly pro-resolving effects during acute inflammatory processes, e.g. in the setting of tissue injury or microbial infection, the consequences of chronic Nrf2 stimulation, for instance in the context of metabolic dysfunction or atherogenic vascular inflammation, remain less well understood. During atherogenesis, Nrf2 can be activated by several mechanisms. Laminar flow and high fluid shear stress have been shown to elicit athero-protective Nrf2-signaling in endothelial cells [83]. This flow-responsive expression of antioxidant genes involves the COX-2-dependent generation of $15 \mathrm{~d}$ PGJ2 for subsequent activation of Nrf2 [82] and suppresses the endothelial cell activation in arteries in vivo [84]. Analogous to this activation by endogenous cyclopentenone-prostaglandins, Nrf2 can also be triggered in endothelial cells and 
macrophages by the electrophilic lipid species present in atherosclerotic plaques [42]. In particular, exposure to OxLDL [103], isolated cyclopentenone-OxPLs $[40,104]$ or to their respective cyclopentenone-isoprostanes $[40,105]$ induces the Nrf2dependent gene expression in vitro [103-105] and in vivo $[40,84,85]$.

The absence of Nrf2 significantly reduces the extent of atherosclerosis in the apolipoprotein-deficient mouse model [106-108]. This phenotype was first reported by Sussan et al., who showed the reduced uptake of modified LDL by Nrf2-deficient macrophages and a decreased expression of scavenger receptor CD36 in the aortic plaques of Nrf2-deficient mice with atherosclerosis [106]. Later studies confirmed this observation, but suggested additional mechanisms by which Nrf2-deficiency might contribute to the atherogenic process. Barajas et al. demonstrated that besides its effects on CD36-mediated lipid uptake and macrophage accumulation in plaques, the lack of Nrf2 also influenced atherogenesis by reducing hepatic cholesterol content and modulating the expression of lipid metabolism genes [107]. Furthermore, a study from our group characterized Nrf2 as an essential regulator of the lipid-induced IL-1 secretion from macrophage-foam cells that drives atherogenic vascular inflammation [108]. The key importance of macrophage-specific Nrf2-signaling for the overall proatherogenic properties of Nrf2 was corroborated by the study of Harada et al., which indicated that Nrf2-activation in macrophages promoted the progression of atherosclerosis by modulating the inflammatory state of macrophages within the atherosclerotic plaques [109].

These studies are in conflict with observations obtained in the LDL receptor-deficient bone marrow chimera model of atherosclerosis. Collins et al. investigated the effects of hematopoietic Nrf2-deficiency after an extensive feeding of a high-cholesterol diet, which also caused severe liver injury. In this model the reduced expression of antioxidant genes in Nrf2-deficient hematopoietic cells resulted in an increased proinflammatory state and aggravated atherogenesis [110]. Ruotsalainen et al. demonstrated the enhanced early atherogenesis in absence of hematopoietic Nrf2 [111]. Their results suggested an increased expression of scavenger receptors and of TLR4, as well as the amplified production of pro-inflammatory MCP-1 and IL-6 as the underlying mechanisms. Collectively, these studies clearly implicate Nrf2dependent gene expression in the pathogenesis of atherosclerosis and suggest that Nrf2-signaling, potentially induced by vascular cyclopentenone-isoprostanes such as EC $[40,42,105]$, relays oxidative stress to the regulation of inflammation. The atheroprotective function of Nrf2-dependent antioxidant response genes has been established in a series of studies that examined the impact of heme-oxygenase 1 
[112], GSH biosynthesis [113], glutathione peroxidase 1 [114] or peroxiredoxins $[115,116]$ on atherogenesis. In addition, the Nrf2-dependent redox regulation appears to support pro-resolving mechanisms during acute inflammation.

Nevertheless, chronic Nrf2-signaling may also exert potent pro-inflammatory effects. For example, exposure to OxPL promotes the differentiation of macrophages towards the pro-inflammatory Mox subset that has been demonstrated in atherosclerotic plaques [117]. Additionally, Nrf2 has been shown to amplify fatty acid-induced IL-1 $\alpha$ production and cholesterol crystal-elicited IL1 $\beta$ secretion, and thereby exacerbates pro-atherogenic, IL-1-driven vascular inflammation [108,118].

Altogether these observations promote the concept that a central function of Nrf2 during inflammation is to regulate the pro-oxidant environment, limit excessive ROS formation and the resulting oxidative injury. The factors that determine whether or not Nrf2 activation conveys overall pro-resolving effects or has pro-inflammatory consequences, as shown for chronic inflammation in atherosclerosis, so far remain unidentified. Therefore, the molecular mechanisms that link the signaling of cyclopentenone-OxPLs via Nrf2 to the either pro-resolving or pro-inflammatory consequences of Nrf2 activation certainly merit further investigation.

\section{Concluding remarks}

In summary, the polyunsaturated fatty acids of membrane phospholipids can be oxidatively modified during inflammatory responses or under conditions of increased oxidative stress. In contrast to the site-specific enzymatic oxidation reactions, that generate the conventional endogenous lipid mediators from phospholipid-derived fatty acids, such radical-mediated oxidation is non-specific and yields a diverse array of structurally and functionally distinct OXPL species. It is now well recognized that these OxPLs are not just inert byproducts of the lipid peroxidation associated with inflammatory conditions, but instead actively influence biological processes. Most OxPL species mediate strong pro-inflammatory effects by triggering pathogen recognition mechanisms that signal microbial infection, and hence activate vigorous innate immune responses. However, OxPLs with anti-inflammatory bioactivities have also been identified. Amongst them, cyclopentenone-containing OxPL and their corresponding cyclopentenone-isoprostanes were found to exhibit powerful antiinflammatory properties and to promote the resolution of inflammation in vivo. In 
particular, OxPL-derived cyclopentenone-isoprostanes mimic the signaling and biological activities of the endogenous cyclopentenone-prostaglandin 15d-PGJ2, a known mediator of resolution. By triggering the Nrf2-signaling pathway, these cyclopentenone-containing lipid mediators efficiently induce key pro-resolving effects, including the inhibition of endothelial activation, the suppression of pro-inflammatory cytokine responses, and the increased clearance of inflammatory infiltrates. Furthermore, compared to the endogenous cyclopentenone-prostaglandin 15d-PGJ2, OxPL-derived cyclopentenone-isoprostanes exhibit a much higher potency; and the structural determinants of their increased bioactivity have been identified [40,51,54].

Thus, by sensing the electrophilic cyclopentenone-OxPL and related isoprostanes that are formed as a result of inflammation-associated lipid peroxidation, the Nrf2pathway appears to modulate the magnitude of oxidative stress and inflammation to support appropriate pro-resolving responses. Still, the chronic activation of Nrf2 by OXPL has been shown to promote the development of a pro-inflammatory macrophage phenotype [117]; and could potentially exacerbate chronic inflammation during atherosclerosis [119]. The nature of the Nrf2-regulated mechanisms that mediate its pro-resolving effects remain to be identified; however these findings clearly establish OxPL-induced Nrf2-signaling as an important pathway linking oxidative stress to the regulation of inflammation. Further investigations into the transport, uptake, signaling and metabolism of cyclopentenone-lipid mediators will be instrumental to delineate the underlying mechanisms and may also identify novel cyclopentenone variants with improved activities and biological profiles. It can be expected that these studies will not only advance our knowledge on the biology of OxPLs and the contribution of cyclopentenones to the resolution of inflammation, but may also discover novel pathways that could be targeted in the therapy of chronic inflammation and infection-associated immunopathologies. 


\section{Acknowledgements}

Work from our laboratory is supported by grants from the Swiss National Science Foundation (SNF152872, SNF157702), the Schweizerische Herzstiftung, the OlgaMayenfisch-Stiftung, the Vontobel-Stiftung, the Fondation Johanna Dürmüller-Bol, the Berne University Research Foundation, the Swiss Lung Association and the $3 R$ Research Foundation Switzerland.

The author declares no financial or commercial conflict of interest.

\section{Figure legends}

Figure 1. Exposure of biological membranes to reactive oxygen species (ROS) generates oxidized phospholipids (OxPLs) with pro-inflammatory or antiinflammatory bioactivities. In the context of inflammation, the polyunsaturated fatty acid side chains of membrane phospholipids can become oxidatively modified. The resulting oxidized phospholipids may exert pro-inflammatory effects by activating pattern recognition receptors (PRRs), including different Toll-like receptors, scavenger receptors, C-reactive protein and natural antibodies. Pattern recognition of OxPLs may result from a direct interaction with their oxidized fatty acids, but can also be mediated by newly formed oxidation-specific epitopes, such as the oxidized phosphocholine moiety or OxPL-protein adducts. Anti-inflammatory OxPLs can inhibit the activation of TLRs by microbial products and interfere with pro-inflammatory NFKB-signaling. Underlying mechanisms are the modification of redox sensitive thiols in IKK $\beta$, of $N F \kappa B$ subunits and of Keap-1 by electrophilic OxPLs or their respective isoprostanes.

\section{Figure 2. Chemical structures of relevant prostaglandins and oxidized}

phospholipids. Prostaglandins and oxidized phospholipids are generated from common precursor phospholipids containing arachidonic acid. The enzymatic generation of prostaglandins involves the release of arachidonic acid from the phospholipid and its subsequent metabolism by cyclooxygenase 1 or 2 and different 
prostaglandin synthases, such as prostaglandin A2 synthase for the generation of PGA2 or prostaglandin D2 synthase, which generates PGD2. PGJ2 and 15-deoxy$\Delta^{12,14}$-PGJ2 are generated by dehydration of PGD2. In contrast, oxidized phospholipids are generated by the non-enzymatic, radical-induced oxidation of arachidonoyl-containing phospholipids. R equals 1-acyl-2-lyso-sn-glycero-3phosphocholine for the oxidized arachidonoyl-containing phosphocholine species. $R$ is $\mathrm{H}$ for the corresponding isoprostanes. PLA2, phospholipase A2; COX, cyclooxygenase; PG, prostaglandin; PL, phospholipid; ROS, reactive oxygen species.

\section{Figure 3. Schematic depiction of Nrf2-activation by pro-resolving} cyclopentenones. Left panel: Under steady state conditions, Nrf2 is bound by its repressor Keap-1. Due to this interaction, Nrf2 is kept inactive and is constantly ubiquitinylated and targeted for proteasomal degradation. Middle panel: In presence of ROS, the polyunsaturated fatty acids of membrane phospholipids are oxidized, resulting the generation of various species of oxidized phospholipids (OxPLs). Fatty acid cyclopentenone-containing isoprostanes, such as EC, can be released from OxPLs by spontaneous or enzymatic deacylation and covalently bind to redoxsensitive cysteine-residues of Keap-1. This results in release of Nrf2, which translocates to the nucleus and initiates transcription of genes containing an antioxidant response element (ARE) in their promoter region. Right panel: Prostaglandin PGD2 is generated from membrane phospholipids containing arachidonic acid by the successive action of phospholipase, cyclooxygenase and prostaglandin D synthase. 15d-PGJ2 is generated from PGD2 as a result of spontaneous dehydration and rearrangement. Analogous to EC, also 15d-PGJ2 activates Nrf2-signaling by modifying redox-sensitive cysteine-residues in Keap-1.

\section{Figure 4. Comparison of the structures of pro-resolving prostaglandin 15-} deoxy- $\Delta^{12,14}-$ PGJ2 and related OxPL-derived isoprostanes. The top row depicts the OxPL-derived epoxyisoprostanes EI, EC and cyclo-EC ordered according to their relative bioactivity. Likely, El gives rise to the cyclopentenone-containing EC, from which the EC-derived lactone cyclo-EC can be formed by an intramolecular reaction between the epoxide and the carboxylic acid. The bottom row shows a comparison between endogenous cyclopentenones PGJ2 and 15d-PGJ2 with EC. Structural 
differences between the molecules as compared to EC are highlighted in blue; the electrophilic enones are indicated by red stars. 


\section{References}

[1] R. Medzhitov, Inflammation 2010: New Adventures of an Old Flame, Cell. 140 (2010) 771-776. doi:10.1016/j.cell.2010.03.006.

[2] O. Takeuchi, S. Akira, Pattern Recognition Receptors and Inflammation, Cell. 140 (2010) 805-820. doi:10.1016/j.cell.2010.01.022.

[3] G.Y. Chen, G. Nuñez, Sterile inflammation: sensing and reacting to damage, Nat. Rev. Immunol. 10 (2010) 826-837. doi:10.1038/nri2873.

[4] C. Nathan, A. Ding, Nonresolving Inflammation, Cell. 140 (2010) 871-882. doi:10.1016/j.cell.2010.02.029.

[5] C.D. Buckley, D.W. Gilroy, C.N. Serhan, Proresolving Lipid Mediators and Mechanisms in the Resolution of Acute Inflammation, Immunity. 40 (2014) 315-327. doi:10.1016/j.immuni.2014.02.009.

[6] C.N. Serhan, Resolution Phase of Inflammation: Novel Endogenous AntiInflammatory and Proresolving Lipid Mediators and Pathways, Annu. Rev. Immunol. 25 (2007) 101-137.

doi:10.1146/annurev.immunol.25.022106.141647.

[7] M. Spite, L.V. Norling, L. Summers, R. Yang, D. Cooper, N.A. Petasis, et al., Resolvin D2 is a potent regulator of leukocytes and controls microbial sepsis, Nature. 461 (2009) 1287-1291. doi:10.1038/nature08541.

[8] J. Walker, E. Dichter, G. Lacorte, D. Kerner, B. Spur, A. Rodriguez, et al., Lipoxin A4 Increases Survival by Decreasing Systemic Inflammation and Bacterial Load in Sepsis, Shock. 36 (2011) 410-416. doi:10.1097/SHK.0b013e31822798c1.

[9] N. Chiang, G. Fredman, F. Bäckhed, S.F. Oh, T. Vickery, B.A. Schmidt, et al., Infection regulates pro-resolving mediators that lower antibiotic requirements, Nature. 484 (2013) 524-528. doi:10.1038/nature11042.

[10] E.J. Haas-Stapleton, Y. Lu, S. Hong, M. Arita, S. Favoreto, S. Nigam, et al., Candida albicans modulates host defense by biosynthesizing the proresolving mediator resolvin E1, PLoS ONE. 2 (2007) e1316. doi:10.1371/journal.pone.0001316.

[11] M. Morita, K. Kuba, A. Ichikawa, M. Nakayama, J. Katahira, R. Iwamoto, et al., The Lipid Mediator Protectin D1 Inhibits Influenza Virus Replication and Improves Severe Influenza, Cell. 153 (2013) 112-125. doi:10.1016/j.cell.2013.02.027.

[12] C.N. Serhan, Pro-resolving lipid mediators are leads for resolution physiology, Nature. 510 (2014) 92-101. doi:10.1038/nature13479.

[13] I. Tabas, C.K. Glass, Anti-inflammatory therapy in chronic disease: challenges and opportunities, Science. 339 (2013) 166-172. doi:10.1126/science.1230720.

[14] H.-J. Kim, S. Ha, H.Y. Lee, K.-J. Lee, ROSics: Chemistry and proteomics of cysteine modifications in redox biology, Mass Spec Rev. 34 (2014) 184208. doi:10.1002/mas.21430.

[15] E. Galkina, K. Ley, Immune and inflammatory mechanisms of atherosclerosis $\left(^{*}\right)$, Annu. Rev. Immunol. 27 (2009) 165-197. doi:10.1146/annurev.immunol.021908.132620.

[16] S. Toyokuni, K. Okamoto, J. Yodoi, H. Hiai, Persistent oxidative stress in cancer, FEBS Lett. 358 (1995) 1-3.

[17] V.N. Bochkov, O.V. Oskolkova, K.G. Birukov, A.-L. Levonen, C.J. Binder, J. Stöckl, Generation and biological activities of oxidized phospholipids, Antioxid. Redox Signal. 12 (2010) 1009-1059. doi:10.1089/ars.2009.2597.

[18] A. Reis, C.M. Spickett, Chemistry of phospholipid oxidation, Biochim. Biophys. Acta. 1818 (2012) 2374-2387. doi:10.1016/j.bbamem.2012.02.002. 
[19] V.N. Bochkov, A. Kadl, J. Huber, F. Gruber, B.R. Binder, N. Leitinger, Protective role of phospholipid oxidation products in endotoxin-induced tissue damage, Nature. 419 (2002) 77-81. doi:10.1038/nature01023.

[20] D. Cruz, A.D. Watson, C.S. Miller, D. Montoya, M.-T. Ochoa, P.A. Sieling, et al., Host-derived oxidized phospholipids and HDL regulate innate immunity in human leprosy, J. Clin. Invest. 118 (2008) 2917-2928. doi:10.1172/JCI34189DS1.

[21] Y. Imai, K. Kuba, G.G. Neely, R. Yaghubian-Malhami, T. Perkmann, G. van Loo, et al., Identification of oxidative stress and Toll-like receptor 4 signaling as a key pathway of acute lung injury, Cell. 133 (2008) 235-249. doi:10.1016/j.cell.2008.02.043.

[22] J.A. Berliner, A.D. Watson, A role for oxidized phospholipids in atherosclerosis, N. Engl. J. Med. 353 (2005) 9-11. doi:10.1056/NEJMp058118.

[23] D. Weismann, K. Hartvigsen, N. Lauer, K.L. Bennett, H.P.N. Scholl, P.C. Issa, et al., Complement factor $\mathrm{H}$ bindsmalondialdehyde epitopes and protectsfrom oxidative stress, Nature. 478 (2012) 76-81.

doi:10.1038/nature10449.

[24] C.R. Stewart, L.M. Stuart, K. Wilkinson, J.M. van Gils, J. Deng, A. Halle, et al., CD36 ligands promote sterile inflammation through assembly of a Tolllike receptor 4 and 6 heterodimer, Nat. Immunol. 11 (2010) 155-161. doi:10.1038/ni.1836.

[25] T.A. Seimon, M.J. Nadolski, X. Liao, J. Magallon, M. Nguyen, N.T. Feric, et al., Atherogenic Lipids and Lipoproteins Trigger CD36-TLR2-Dependent Apoptosis in Macrophages Undergoing Endoplasmic Reticulum Stress, Cell Metabolism. 12 (2010) 467-482. doi:10.1016/j.cmet.2010.09.010.

[26] E.A. Podrez, Identification of a Novel Family of Oxidized Phospholipids That Serve as Ligands for the Macrophage Scavenger Receptor CD36, Journal of Biological Chemistry. 277 (2002) 38503-38516. doi:10.1074/jbc.M203318200.

[27] K. Gillotte-Taylor, A. Boullier, J.L. Witztum, D. Steinberg, O. Quehenberger, Scavenger receptor class $B$ type I as a receptor for oxidized low density lipoprotein, The Journal of Lipid Research. 42 (2001) 1474-1482.

[28] I. Shechter, A.M. Fogelman, M.E. Haberland, J. Seager, M. Hokom, P.A. Edwards, The metabolism of native and malondialdehyde-altered low density lipoproteins by human monocyte-macrophages, The Journal of Lipid Research. 22 (1981) 63-71.

[29] M.-K. Chang, C.J. Binder, M. Torzewski, J.L. Witztum, C-reactive protein binds to both oxidized LDL and apoptotic cells through recognition of a common ligand: Phosphorylcholine of oxidized phospholipids, Proc. Natl. Acad. Sci. U.S.a. 99 (2002) 13043-13048. doi:10.1073/pnas.192399699.

[30] W. Palinski, S. Hörkkö, E. Miller, U.P. Steinbrecher, H.C. Powell, L.K. Curtiss, et al., Cloning of monoclonal autoantibodies to epitopes of oxidized lipoproteins from apolipoprotein E-deficient mice. Demonstration of epitopes of oxidized low density lipoprotein in human plasma, J. Clin. Invest. 98 (1996) 800-814. doi:10.1172/JCl118853.

[31] M.-Y. Chou, L. Fogelstrand, K. Hartvigsen, L.F. Hansen, D. Woelkers, P.X. Shaw, et al., Oxidation-specific epitopes are dominant targets of innate natural antibodies in mice and humans, J. Clin. Invest. 119 (2009) 13351349. doi:10.1172/JCI36800.

[32] F.J. Sheedy, A. Grebe, K.J. Rayner, P. Kalantari, B. Ramkhelawon, S.B. Carpenter, et al., CD36 coordinates NLRP3 inflammasome activation by facilitating intracellular nucleation of soluble ligands into particulate ligands in sterile inflammation, Nat. Immunol. 14 (2013) 812-820.

doi:10.1038/ni.2639. 
[33] A. Kadl, P.R. Sharma, W. Chen, R. Agrawal, A.K. Meher, S. Rudraiah, et al., Free Radical Biology \& Medicine, Free Radic. Biol. Med. 51 (2011) 1903-1909. doi:10.1016/j.freeradbiomed.2011.08.026.

[34] D. Weismann, C.J. Binder, Biochimica et Biophysica Acta, BBA Biomembranes. 1818 (2012) 2465-2475. doi:10.1016/j.bbamem.2012.01.018.

[35] C.J. Binder, S. Hörkkö, A. Dewan, M.-K. Chang, E.P. Kieu, C.S. Goodyear, et al., Pneumococcal vaccination decreases atherosclerotic lesion formation: molecular mimicry between Streptococcus pneumoniae and oxidized LDL, Nat. Med. 9 (2003) 736-743. doi:10.1038/nm876.

[36] N. Leitinger, T.R. Tyner, L. Oslund, C. Rizza, G. Subbanagounder, H. Lee, et al., Structurally similar oxidized phospholipids differentially regulate endothelial binding of monocytes and neutrophils, Proc. Natl. Acad. Sci. U.S.a. 96 (1999) 12010-12015.

[37] Z. Ma, J. Li, L. Yang, Y. Mu, W. Xie, B. Pitt, et al., Inhibition of LPS- and CpG DNA-induced TNF-alpha response by oxidized phospholipids, Am. J. Physiol. Lung Cell Mol. Physiol. 286 (2004) L808-16. doi:10.1152/ajplung.00220.2003.

[38] S. Bluml, S. Kirchberger, V.N. Bochkov, G. Kronke, K. Stuhlmeier, O. Majdic, et al., Oxidized Phospholipids Negatively Regulate Dendritic Cell Maturation Induced by TLRs and CD40, The Journal of Immunology. 175 (2005) 501-508. doi:10.4049/jimmunol.175.1.501.

[39] C. Erridge, S. Kennedy, C.M. Spickett, D.J. Webb, Oxidized Phospholipid Inhibition of Toll-like Receptor (TLR) Signaling Is Restricted to TLR2 and TLR4: ROLES FOR CD14, LPS-BINDING PROTEIN, AND MD2 AS TARGETS FOR SPECIFICITY OF INHIBITION, Journal of Biological Chemistry. 283 (2008) 24748-24759. doi:10.1074/jbc.M800352200.

[40] P. Bretscher, J. Egger, A. Shamshiev, M. Trötzmüller, H. Köfeler, E.M. Carreira, et al., Phospholipid oxidation generates potent anti-inflammatory lipid mediators that mimic structurally related pro-resolving eicosanoids by activating Nrf2, EMBO Molecular Medicine. 7 (2015) 593-607. doi:10.15252/emmm.201404702.

[41] A.D. Watson, N. Leitinger, M. Navab, K.F. Faull, S. Hörkkö, J.L. Witztum, et al., Structural identification by mass spectrometry of oxidized phospholipids in minimally oxidized low density lipoprotein that induce monocyte/endothelial interactions and evidence for their presence in vivo, J. Biol. Chem. 272 (1997) 13597-13607.

[42] A.D. Watson, G. Subbanagounder, D.S. Welsbie, K.F. Faull, M. Navab, M.E. Jung, et al., Structural identification of a novel pro-inflammatory epoxyisoprostane phospholipid in mildly oxidized low density lipoprotein, J. Biol. Chem. 274 (1999) 24787-24798.

[43] G. Subbanagounder, Y. Deng, C. Borromeo, A.N. Dooley, J.A. Berliner, R.G. Salomon, Hydroxy alkenal phospholipids regulate inflammatory functions of endothelial cells, Vascul. Pharmacol. 38 (2002) 201-209.

[44] R. Li, K.P. Mouillesseaux, D. Montoya, D. Cruz, N. Gharavi, M. Dun, et al., Identification of prostaglandin E2 receptor subtype 2 as a receptor activated by OxPAPC, Circ. Res. 98 (2006) 642-650. doi:10.1161/01.RES.0000207394.39249.fc.

[45] H. Lee, W. Shi, P. Tontonoz, S. Wang, G. Subbanagounder, C.C. Hedrick, et al., Role for peroxisome proliferator-activated receptor alpha in oxidized phospholipid-induced synthesis of monocyte chemotactic protein-1 and interleukin-8 by endothelial cells, Circ. Res. 87 (2000) 516-521.

[46] G. Subbanagounder, J.W. Wong, H. Lee, K.F. Faull, E. Miller, J.L. Witztum, et al., Epoxyisoprostane and epoxycyclopentenone phospholipids regulate monocyte chemotactic protein-1 and interleukin- 8 synthesis. Formation of 
these oxidized phospholipids in response to interleukin-1beta, J. Biol. Chem. 277 (2002) 7271-7281. doi:10.1074/jbc.M107602200.

[47] O.V. Oskolkova, T. Afonyushkin, B. Preinerstorfer, W. Bicker, E. von Schlieffen, E. Hainzl, et al., Oxidized Phospholipids Are More Potent Antagonists of Lipopolysaccharide than Inducers of Inflammation, The Journal of Immunology. 185 (2010) 7706-7712.

doi:10.4049/jimmunol.0903594.

[48] K.G. Birukov, V.N. Bochkov, A.A. Birukova, K. Kawkitinarong, A. Rios, A. Leitner, et al., Epoxycyclopentenone-containing oxidized phospholipids restore endothelial barrier function via Cdc42 and Rac, Circ. Res. 95 (2004) 892-901. doi:10.1161/01.RES.0000147310.18962.06.

[49] R.K. Thimmulappa, X. Gang, J.-H. Kim, T.E. Sussan, J.L. Witztum, S. Biswal, Biochemical and Biophysical Research Communications, Biochem. Biophys. Res. Commun. 426 (2012) 253-259.

doi:10.1016/j.bbrc.2012.08.076.

[50] J. Egger, P. Bretscher, S. Freigang, M. Kopf, E.M. Carreira, Synthesis of epoxyisoprostanes: effects in reducing secretion of pro-inflammatory cytokines IL-6 and IL-12, Angew. Chem. Int. Ed. Engl. 52 (2013) 53825385. doi:10.1002/anie.201300739.

[51] J. Egger, P. Bretscher, S. Freigang, M. Kopf, E.M. Carreira, Discovery of a highly potent anti-inflammatory epoxyisoprostane-derived lactone, J. Am. Chem. Soc. 136 (2014) 17382-17385. doi:10.1021/ja509892u.

[52] J. Choi, W. Zhang, X. Gu, X. Chen, L. Hong, J.M. Laird, et al., Lysophosphatidylcholine is generated by spontaneous deacylation of oxidized phospholipids, Chem. Res. Toxicol. 24 (2011) 111-118. doi:10.1021/tx100305b.

[53] T. Suzuki, M. Yamamoto, Free Radical Biology and Medicine, Free Radic. Biol. Med. 88 (2015) 93-100. doi:10.1016/j.freeradbiomed.2015.06.006.

[54] J. Egger, S. Fischer, P. Bretscher, S. Freigang, M. Kopf, E.M. Carreira, Total Synthesis of Prostaglandin 15d-PGJ(2) and Investigation of its Effect on the Secretion of IL-6 and IL-12, Org. Lett. 17 (2015) 4340-4343. doi:10.1021/acs.orglett.5b02181.

[55] A.L. Groeger, C. Cipollina, M.P. Cole, S.R. Woodcock, G. Bonacci, T.K. Rudolph, et al., Cyclooxygenase-2 generates anti-inflammatory mediators from omega-3 fatty acids, Nat. Chem. Biol. 6 (2010) 433-441. doi:10.1038/nchembio.367.

[56] C. Cipollina, Endogenous Generation and Signaling Actions of Omega-3 Fatty Acid Electrophilic Derivatives, Biomed Res Int. 2015 (2015) 501792. doi:10.1155/2015/501792.

[58] A. Rossi, P. Kapahi, G. Natoli, T. Takahashi, Y. Chen, M. Karin, et al., Antiinflammatory cyclopentenone prostaglandins are direct inhibitors of IkappaB kinase, Nature. 403 (2000) 103-108. doi:10.1038/47520.

[59] A. Castrillo, M.J. Díaz-Guerra, S. Hortelano, P. Martín-Sanz, L. Bosca, Inhibition of IkappaB kinase and IkappaB phosphorylation by 15-deoxyDelta(12,14)-prostaglandin $\mathrm{J}(2)$ in activated murine macrophages, Molecular and Cellular Biology. 20 (2000) 1692-1698.

[60] T. Kato, M. Fukushima, S. Kurozumi, R. Noyori, Antitumor activity of delta 7-prostaglandin A1 and delta 12-prostaglandin $\mathrm{J} 2$ in vitro and in vivo, Cancer Res. 46 (1986) 3538-3542.

[61] M. Suzuki, T. Kiho, K. Tomokiyo, K. Furuta, S. Fukushima, Y. Takeuchi, et al., Rational design of antitumor prostaglandins with high biological stability, J. Med. Chem. 41 (1998) 3084-3090. doi:10.1021/jm9801657.

[62] H. Wang, M. Linetsky, J. Guo, J. Choi, L. Hong, A.S. Chamberlain, et al., 4Hydroxy-7-oxo-5-heptenoic Acid (HOHA) Lactone is a Biologically Active Precursor for the Generation of 2-( $\omega$-Carboxyethyl)pyrrole (CEP) 
Derivatives of Proteins and Ethanolamine Phospholipids, Chem. Res.

Toxicol. 28 (2015) 967-977. doi:10.1021/acs.chemrestox.5b00001.

[63] E. Kansanen, A.M. Kivelä, A.-L. Levonen, Free Radical Biology \& Medicine, Free Radic. Biol. Med. 47 (2009) 1310-1317.

doi:10.1016/j.freeradbiomed.2009.06.030.

[64] D.S. Straus, C.K. Glass, Cyclopentenone prostaglandins: new insights on biological activities and cellular targets, Med Res Rev. 21 (2001) 185-210.

[65] Y.-J. Surh, H.-K. Na, J.-M. Park, H.-N. Lee, W. Kim, I.-S. Yoon, et al., Biochemical Pharmacology, Biochemical Pharmacology. 82 (2011) 13351351. doi:10.1016/j.bcp.2011.07.100.

[66] C. Jiang, A.T. Ting, B. Seed, PPAR-gamma agonists inhibit production of monocyte inflammatory cytokines, Nature. 391 (1998) 82-86. doi:10.1038/34184.

[67] M. Ricote, A.C. Li, T.M. Willson, C.J. Kelly, C.K. Glass, The peroxisome proliferator-activated receptor-gamma is a negative regulator of macrophage activation, Nature. 391 (1998) 79-82. doi:10.1038/34178.

[68] B.M. Forman, P. Tontonoz, J. Chen, R.P. Brun, B.M. Spiegelman, R.M. Evans, 15-Deoxy-delta 12, 14-prostaglandin $\mathrm{J} 2$ is a ligand for the adipocyte determination factor PPAR gamma, Cell. 83 (1995) 803-812.

[69] S.A. Kliewer, J.M. Lenhard, T.M. Willson, I. Patel, D.C. Morris, J.M. Lehmann, A prostaglandin $\mathrm{J} 2$ metabolite binds peroxisome proliferatoractivated receptor gamma and promotes adipocyte differentiation, Cell. 83 (1995) 813-819.

[70] A. Chawla, Y. Barak, L. Nagy, D. Liao, P. Tontonoz, R.M. Evans, PPARgamma dependent and independent effects on macrophage-gene expression in lipid metabolism and inflammation, Nat. Med. 7 (2001) 4852. doi:10.1038/83336.

[71] D.S. Straus, G. Pascual, M. Li, J.S. Welch, M. Ricote, C.H. Hsiang, et al., 15-deoxy-delta 12,14-prostaglandin J2 inhibits multiple steps in the NFkappa B signaling pathway, Proc. Natl. Acad. Sci. U.S.a. 97 (2000) 48444849.

[72] E. Cernuda-Morollón, E. Pineda-Molina, F.J. Cañada, D. Pérez-Sala, 15Deoxy-Delta 12,14-prostaglandin J2 inhibition of NF-kappaB-DNA binding through covalent modification of the p50 subunit, J. Biol. Chem. 276 (2001) 35530-35536. doi:10.1074/jbc.M104518200.

[73] K. Itoh, T. Chiba, S. Takahashi, T. Ishii, K. Igarashi, Y. Katoh, et al., An Nrf2/small Maf heterodimer mediates the induction of phase II detoxifying enzyme genes through antioxidant response elements, Biochem. Biophys. Res. Commun. 236 (1997) 313-322.

[74] A.-L. Levonen, A. Landar, A. Ramachandran, E.K. Ceaser, D.A. Dickinson, G. Zanoni, et al., Cellular mechanisms of redox cell signalling: role of cysteine modification in controlling antioxidant defences in response to electrophilic lipid oxidation products, Biochem. J. 378 (2004) 373-382. doi:10.1042/BJ20031049.

[75] D.W. Gilroy, P.R. Colville-Nash, D. Willis, J. Chivers, M.J. Paul-Clark, D.A. Willoughby, Inducible cyclooxygenase may have anti-inflammatory properties, Nat. Med. 5 (1999) 698-701. doi:10.1038/9550.

[76] Y. Urade, M. Ujihara, Y. Horiguchi, K. Ikai, O. Hayaishi, The major source of endogenous prostaglandin D2 production is likely antigen-presenting cells. Localization of glutathione-requiring prostaglandin D synthetase in histiocytes, dendritic, and Kupffer cells in various rat tissues, J. Immunol. 143 (1989) 2982-2989.

[77] Y. Taba, T. Sasaguri, M. Miyagi, T. Abumiya, Y. Miwa, T. Ikeda, et al., Fluid shear stress induces lipocalin-type prostaglandin $\mathrm{D}(2)$ synthase expression in vascular endothelial cells, Circ. Res. 86 (2000) 967-973. 
[78] R. Rajakariar, M. Hilliard, T. Lawrence, S. Trivedi, P. Colville-Nash, G. Bellingan, et al., Hematopoietic prostaglandin D2 synthase controls the onset and resolution of acute inflammation through PGD2 and 15-

deoxyDelta12 14 PGJ2, Proceedings of the National Academy of Sciences. 104 (2007) 20979-20984. doi:10.1073/pnas.0707394104.

[79] H. Zhu, M. Xia, M. Hou, Z. Tang, Y. Li, J. Ma, et al., Ox-LDL plays dual effect in modulating expression of inflammatory molecules through LOX-1 pathway in human umbilical vein endothelial cells, Front. Biosci. 10 (2005) 2585-2594.

[80] J. Kaplan, J.A. Cook, M. O'Connor, B. Zingarelli, Peroxisome proliferatoractivated receptor gamma is required for the inhibitory effect of ciglitazone but not 15-deoxy-Delta 12,14-prostaglandin J2 on the NFkappaB pathway in human endothelial cells, Shock. 28 (2007) 722-726. doi:10.1097/SHK.0b013e318055683a.

[81] R. Prasad, S. Giri, A.K. Singh, I. Singh, 15-deoxy-delta12,14-prostaglandin J2 attenuates endothelial-monocyte interaction: implication for inflammatory diseases, J Inflamm. 5 (2008) 14. doi:10.1186/1476-9255-514.

[82] T. Hosoya, A. Maruyama, M.-I. Kang, Y. Kawatani, T. Shibata, K. Uchida, et al., Differential responses of the Nrf2-Keap1 system to laminar and oscillatory shear stresses in endothelial cells, J. Biol. Chem. 280 (2005) 27244-27250. doi:10.1074/jbc.M502551200.

[83] X.-L. Chen, S.E. Varner, A.S. Rao, J.Y. Grey, S. Thomas, C.K. Cook, et al., Laminar flow induction of antioxidant response element-mediated genes in endothelial cells. A novel anti-inflammatory mechanism, J. Biol. Chem. 278 (2003) 703-711. doi:10.1074/jbc.M203161200.

[84] M. Zakkar, K. Van der Heiden, L.A. Luong, H. Chaudhury, S. Cuhlmann, S.S. Hamdulay, et al., Activation of Nrf2 in endothelial cells protects arteries from exhibiting a proinflammatory state, Arterioscler. Thromb. Vasc. Biol. 29 (2009) 1851-1857. doi:10.1161/ATVBAHA.109.193375.

[85] H.-K. Jyrkkänen, E. Kansanen, M. Inkala, A.M. Kivelä, H. Hurttila, S.E. Heinonen, et al., Nrf2 regulates antioxidant gene expression evoked by oxidized phospholipids in endothelial cells and murine arteries in vivo, Circ. Res. 103 (2008) e1-9. doi:10.1161/CIRCRESAHA.108.176883.

[86] S. Marzocco, R. Di Paola, E. Mazzon, T. Genovese, D. Britti, A. Pinto, et al., The cyclopentenone prostaglandin 15-deoxydelta(12,14)-prostaglandin $\mathrm{J} 2$ attenuates the development of zymosan-induced shock, Intensive Care Med. 31 (2005) 693-700. doi:10.1007/s00134-005-2596-2.

[87] M.A. Monroy, K.K. Opperman, M. Pucciarelli, S. Yerrum, D.A. Berg, J.M. Daly, THE PPARgamma ligand 15d-PGJ2 modulates macrophage activation after injury in a murine trauma model, Shock. 28 (2007) 186191. doi:10.1097/shk.0b013e3180310982.

[88] K. Itoh, M. Mochizuki, Y. Ishii, T. Ishii, T. Shibata, Y. Kawamoto, et al., Transcription Factor Nrf2 Regulates Inflammation by Mediating the Effect of 15-Deoxy-12,14-Prostaglandin J2, Molecular and Cellular Biology. 24 (2004) 36-45. doi:10.1128/MCB.24.1.36-45.2004.

[89] M. Mochizuki, Y. Ishii, K. Itoh, T. lizuka, Y. Morishima, T. Kimura, et al., Role of 15-Deoxy $\Delta$ 12,14Prostaglandin J 2and Nrf2 Pathways in Protection against Acute Lung Injury, Am J Respir Crit Care Med. 171 (2005) 12601266. doi:10.1164/rccm.200406-7550C.

[90] D.W. Gilroy, P.R. Colville-Nash, S. McMaster, D.A. Sawatzky, D.A. Willoughby, T. Lawrence, Inducible cyclooxygenase-derived 15deoxy(Delta)12-14PGJ2 brings about acute inflammatory resolution in rat pleurisy by inducing neutrophil and macrophage apoptosis, The FASEB Journal. 17 (2003) 2269-2271. doi:10.1096/fj.02-1162fje. 
[91] S. Hortelano, A. Castrillo, A.M. Alvarez, L. Bosca, Contribution of Cyclopentenone Prostaglandins to the Resolution of Inflammation Through the Potentiation of Apoptosis in Activated Macrophages, The Journal of Immunology. 165 (2000) 6525-6531. doi:10.4049/jimmunol.165.11.6525.

[92] C. Ward, I. Dransfield, J. Murray, S.N. Farrow, C. Haslett, A.G. Rossi, Prostaglandin D2 and Its Metabolites Induce Caspase-Dependent Granulocyte Apoptosis That Is Mediated Via Inhibition of I B Degradation Using a Peroxisome Proliferator-Activated Receptor- -Independent Mechanism, The Journal of Immunology. 168 (2002) 6232-6243. doi:10.4049/jimmunol.168.12.6232.

[93] S.G. Trivedi, J. Newson, R. Rajakariar, T.S. Jacques, R. Hannon, Y. Kanaoka, et al., Essential role for hematopoietic prostaglandin D2 synthase in the control of delayed type hypersensitivity, Proc. Natl. Acad. Sci. U.S.a. 103 (2006) 5179-5184. doi:10.1073/pnas.0507175103.

[94] R.K. Thimmulappa, C. Scollick, K. Traore, M. Yates, M.A. Trush, K.T. Liby, et al., Nrf2-dependent protection from LPS induced inflammatory response and mortality by CDDO-Imidazolide, Biochem. Biophys. Res. Commun. 351 (2006) 883-889. doi:10.1016/j.bbrc.2006.10.102.

[95] X. Kong, R. Thimmulappa, F. Craciun, C. Harvey, A. Singh, P. Kombairaju, et al., Enhancing Nrf2 pathway by disruption of Keap1 in myeloid leukocytes protects against sepsis, Am J Respir Crit Care Med. 184 (2011) 928-938. doi:10.1164/rccm.201102-02710C.

[96] J. Athale, A. Ulrich, N.C. MacGarvey, R.R. Bartz, K.E. Welty-Wolf, H.B. Suliman, et al., Nrf2 promotes alveolar mitochondrial biogenesis and resolution of lung injury in Staphylococcus aureus pneumonia in mice, Free Radic. Biol. Med. 53 (2012) 1584-1594. doi:10.1016/j.freeradbiomed.2012.08.009.

[97] A.A. Lugade, R.R. Vethanayagam, M. Nasirikenari, P.N. Bogner, B.H. Segal, Y. Thanavala, Nrf2 regulates chronic lung inflammation and B-cell responses to nontypeable Haemophilus influenzae, Am J Respir Cell Mol Biol. 45 (2011) 557-565. doi:10.1165/rcmb.2010-03210C.

[98] N. Keleku-Lukwete, M. Suzuki, A. Otsuki, K. Tsuchida, S. Katayama, M. Hayashi, et al., Amelioration of inflammation and tissue damage in sickle cell model mice by Nrf2 activation, Proceedings of the National Academy of Sciences. 112 (2015) 12169-12174. doi:10.1073/pnas.1509158112.

[99] D. Olagnier, S. Peri, C. Steel, N. van Montfoort, C. Chiang, V. Beljanski, et al., Cellular oxidative stress response controls the antiviral and apoptotic programs in dengue virus-infected dendritic cells, PLoS Pathog. 10 (2014) e1004566. doi:10.1371/journal.ppat.1004566.

[100] H.-Y. Cho, F. Imani, L. Miller-DeGraff, D. Walters, G.A. Melendi, M. Yamamoto, et al., Antiviral activity of Nrf2 in a murine model of respiratory syncytial virus disease, Am J Respir Crit Care Med. 179 (2009) 138-150. doi:10.1164/rccm.200804-5350C.

[101] A. Page, V.A. Volchkova, Saint Patrick Reid, M. Mateo, A. Bagnaud-Baule, K. Nemirov, et al., Marburgvirus Hijacks Nrf2-Dependent Pathway by Targeting Nrf2-Negative Regulator Keap1, CellReports. 6 (2014) 10261036. doi:10.1016/j.celrep.2014.02.027.

[102] N. Komaravelli, B. Tian, T. Ivanciuc, N. Mautemps, A.R. Brasier, R.P. Garofalo, et al., Free Radical Biology and Medicine, Free Radic. Biol. Med. 88 (2015) 391-403. doi:10.1016/j.freeradbiomed.2015.05.043.

[103] T. Ishii, K. Itoh, E. Ruiz, D.S. Leake, H. Unoki, M. Yamamoto, et al., Role of $\mathrm{Nrf2}$ in the regulation of CD36 and stress protein expression in murine macrophages: activation by oxidatively modified LDL and 4-

hydroxynonenal, Circ. Res. 94 (2004) 609-616.

doi:10.1161/01.RES.0000119171.44657.45. 
[104] R. Li, W. Chen, R. Yanes, S. Lee, J.A. Berliner, OKL38 is an oxidative stress response gene stimulated by oxidized phospholipids, The Journal of Lipid Research. 48 (2006) 709-715. doi:10.1194/jlr.M600501-JLR200.

[105] X. Yan, S. Lee, B.G. Gugiu, L. Koroniak, M.E. Jung, J. Berliner, et al., Biochemical and Biophysical Research Communications, Biochem.

Biophys. Res. Commun. 444 (2014) 69-74.

doi:10.1016/j.bbrc.2014.01.016.

[106] T.E. Sussan, J. Jun, R. Thimmulappa, D. Bedja, M. Antero, K.L.

Gabrielson, et al., Disruption of Nrf2, a key inducer of antioxidant defenses, attenuates ApoE-mediated atherosclerosis in mice, PLoS ONE. 3 (2008) e3791. doi:10.1371/journal.pone.0003791.

[107] B. Barajas, N. Che, F. Yin, A. Rowshanrad, L.D. Orozco, K.W. Gong, et al., NF-E2-related factor 2 promotes atherosclerosis by effects on plasma lipoproteins and cholesterol transport that overshadow antioxidant protection, Arterioscler. Thromb. Vasc. Biol. 31 (2011) 58-66. doi:10.1161/ATVBAHA.110.210906.

[108] S. Freigang, F. Ampenberger, G. Spohn, S. Heer, A.T. Shamshiev, J. Kisielow, et al., Nrf2 is essential for cholesterol crystal-induced inflammasome activation and exacerbation of atherosclerosis, Eur. J. Immunol. 41 (2011) 2040-2051. doi:10.1002/eji.201041316.

[109] N. Harada, K. Ito, T. Hosoya, J. Mimura, A. Maruyama, N. Noguchi, et al., Nrf2 in bone marrow-derived cells positively contributes to the advanced stage of atherosclerotic plaque formation, Free Radic. Biol. Med. 53 (2012) 2256-2262. doi:10.1016/j.freeradbiomed.2012.10.001.

[110] A.R. Collins, A.A. Gupte, R. Ji, M.R. Ramirez, L.J. Minze, J.Z. Liu, et al., Myeloid deletion of nuclear factor erythroid 2-related factor 2 increases atherosclerosis and liver injury, Arterioscler. Thromb. Vasc. Biol. 32 (2012) 2839-2846. doi:10.1161/ATVBAHA.112.300345.

[111] A.-K. Ruotsalainen, M. Inkala, M.E. Partanen, J.P. Lappalainen, E. Kansanen, P.I. Mäkinen, et al., The absence of macrophage Nrf2 promotes early atherogenesis, Cardiovascular Research. 98 (2013) 107-115. doi:10.1093/cvr/cvt008.

[112] S.H. Juan, T.S. Lee, K.W. Tseng, J.Y. Liou, S.K. Shyue, K.K. Wu, et al., Adenovirus-mediated heme oxygenase-1 gene transfer inhibits the development of atherosclerosis in apolipoprotein E-deficient mice, Circulation. 104 (2001) 1519-1525.

[113] M. Rosenblat, R. Coleman, M. Aviram, Increased macrophage glutathione content reduces cell-mediated oxidation of LDL and atherosclerosis in apolipoprotein E-deficient mice, Atherosclerosis. 163 (2002) 17-28.

[114] M. Torzewski, V. Ochsenhirt, A.L. Kleschyov, M. Oelze, A. Daiber, H. Li, et al., Deficiency of glutathione peroxidase-1 accelerates the progression of atherosclerosis in apolipoprotein E-deficient mice, Arterioscler. Thromb. Vasc. Biol. 27 (2007) 850-857. doi:10.1161/01.ATV.0000258809.47285.07.

[115] J. Kisucka, A.K. Chauhan, I.S. Patten, A. Yesilaltay, C. Neumann, R.A. Van Etten, et al., Peroxiredoxin1 prevents excessive endothelial activation and early atherosclerosis, Circ. Res. 103 (2008) 598-605. doi:10.1161/CIRCRESAHA.108.174870.

[116] J.-G. Park, J.-Y. Yoo, S.-J. Jeong, J.-H. Choi, M.-R. Lee, M.-N. Lee, et al., Peroxiredoxin 2 deficiency exacerbates atherosclerosis in apolipoprotein Edeficient mice, Circ. Res. 109 (2011) 739-749. doi:10.1161/CIRCRESAHA.111.245530.

[117] A. Kadl, A.K. Meher, P.R. Sharma, M.Y. Lee, A.C. Doran, S.R. Johnstone, et al., Identification of a novel macrophage phenotype that develops in response to atherogenic phospholipids via Nrf2, Circ. Res. 107 (2010) 
737-746. doi:10.1161/CIRCRESAHA.109.215715.

[118] S. Freigang, F. Ampenberger, A. Weiss, T.-D. Kanneganti, Y. Iwakura, M. Hersberger, et al., Fatty acid-induced mitochondrial uncoupling elicits inflammasome-independent IL-1 $\alpha$ and sterile vascular inflammation in atherosclerosis, Nat. Immunol. 14 (2013) 1045-1053. doi:10.1038/ni.2704.

[119] J. Mimura, K. Itoh, Role of Nrf2 in the pathogenesis of atherosclerosis, Free Radic. Biol. Med. 88 (2015) 221-232. doi:10.1016/j.freeradbiomed.2015.06.019. 
microbial infection

\section{Inflammation}

tissue injury

metabolic dysfunction

Oxidative stress

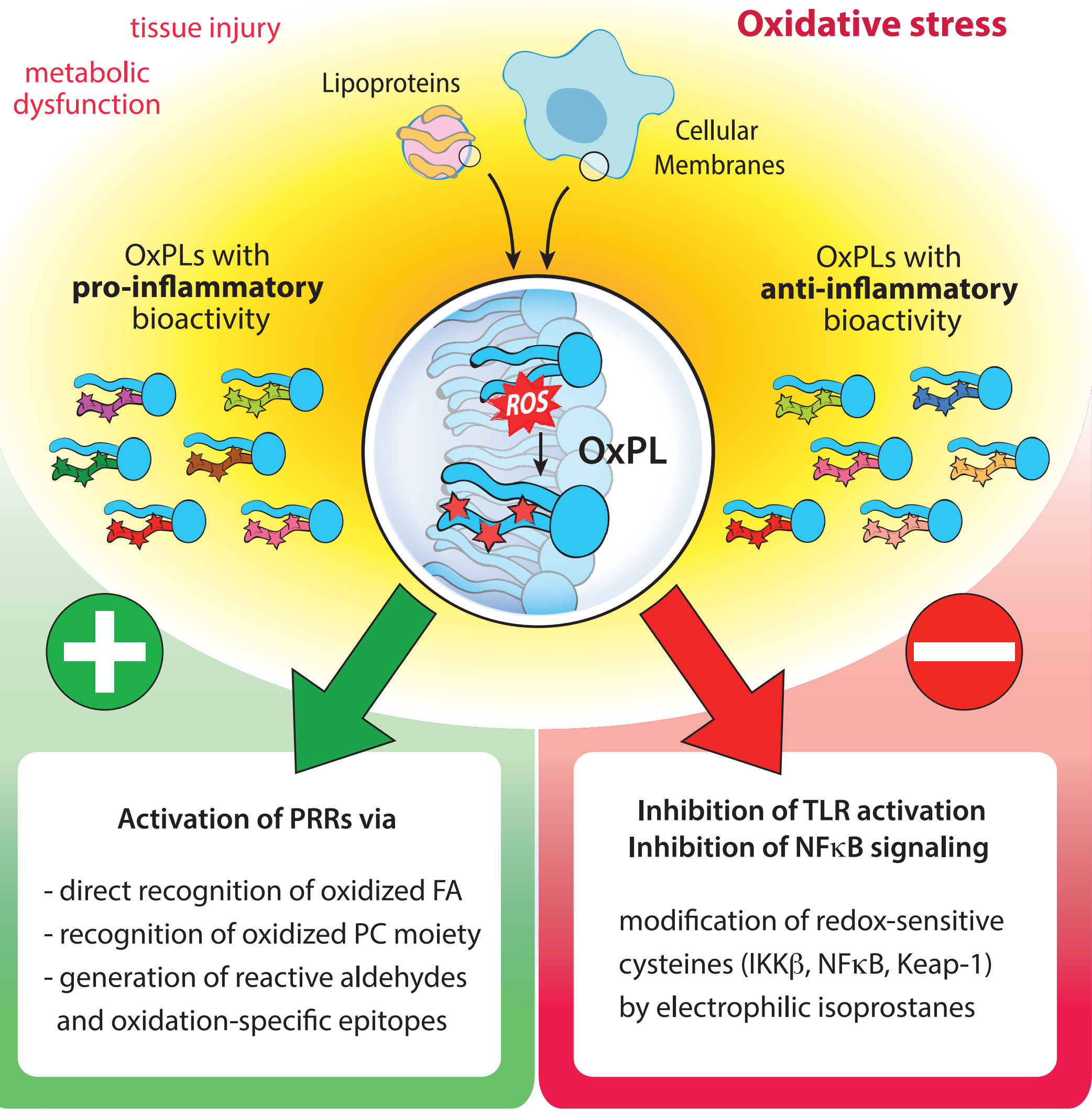


PLA2, COX

PG synthases

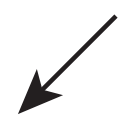

PGA2<smiles>CCCCCC(O)/C=C/C1C(=O)CC(O)C1C/C=C/CCCC(=O)O</smiles>

PGD2<smiles>CCCCCC(O)/C=C/C1C(=O)C=CC1C/C=C/CCCC(=O)O</smiles>

PGJ2

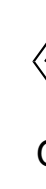

$\overbrace{\mathrm{O}}^{C}$

$\Delta^{12-P G J 2}$

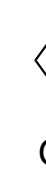<smiles>CCCCC/C=C/C=C1/C(=O)C=CC1C/C=C/CCCC(=O)O</smiles>

15-deoxy- $\Delta^{12,14-P G J 2 ~}$<smiles>[R]OC(=O)CCCC(=O)O</smiles>

Glutaroyl (G)-PL<smiles>[R]OC(=O)CCCC(=O)C=CC(=O)O</smiles>

5-keto-6-octene-dioyl (KOdiA)-PL<smiles>[R]OC(=O)CCCC1OC1C=C1C(=O)CC(O)C1CC=CCCCCC</smiles>

5,6-epoxyisoprostane E2 (EI)-PL<smiles>[R]OC(=O)CCCC1OC1/C=C1/C(=O)C=CC1C/C=C/CCCCC</smiles>

5,6-epoxyisoprostane A2 (EC)-PL 
Steady state

Activation by OxPLs

Activation by $15 \mathrm{~d}-\mathrm{PGJ} 2$

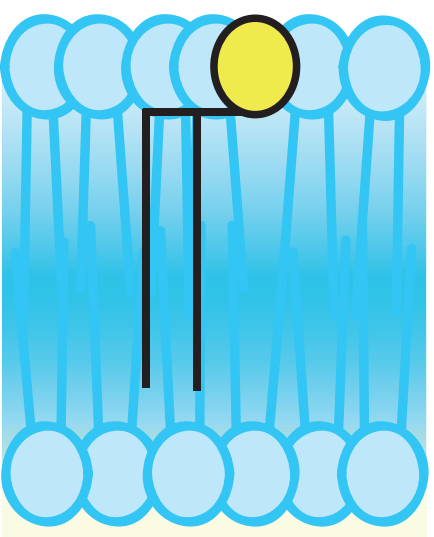

Inactive Nrf2
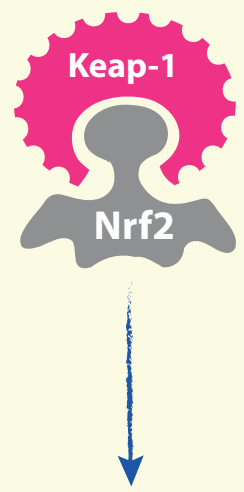

Proteasomal degradation

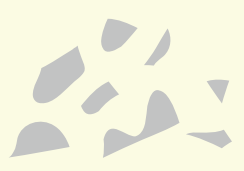

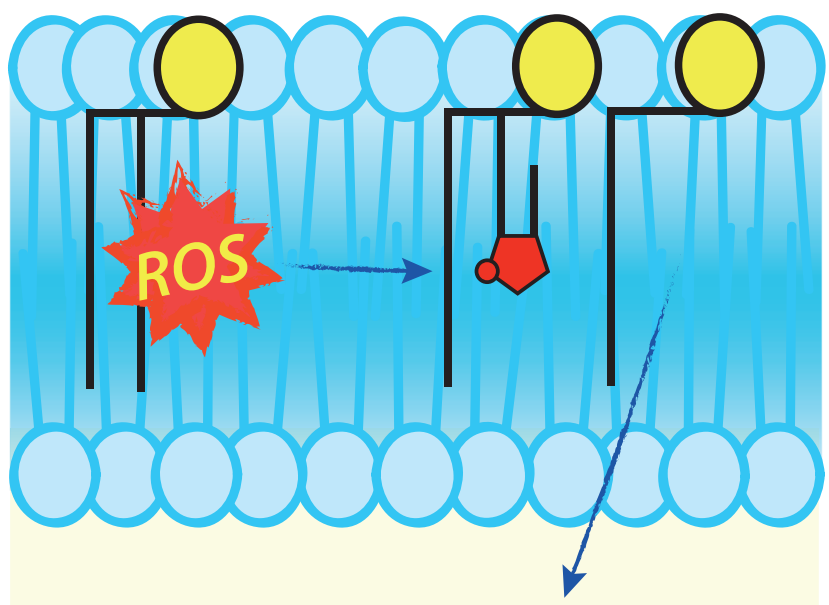
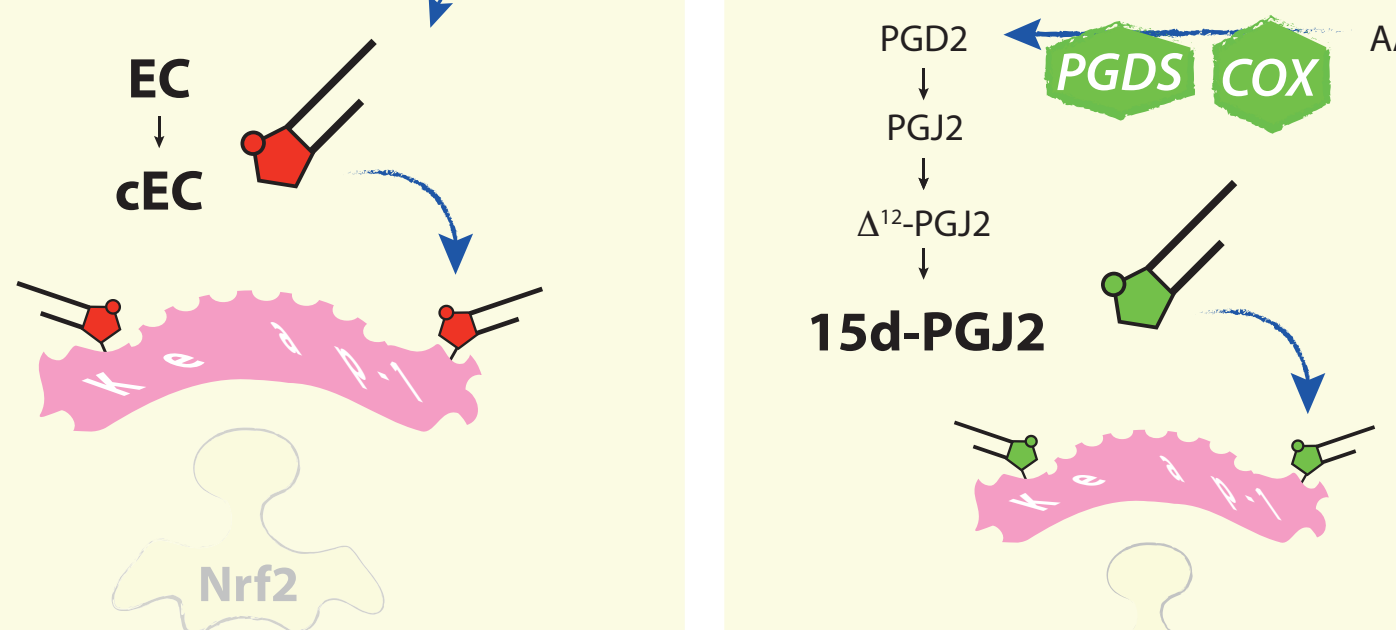

Nrf2

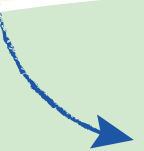

Active Nrf2

Transcription of target genes:

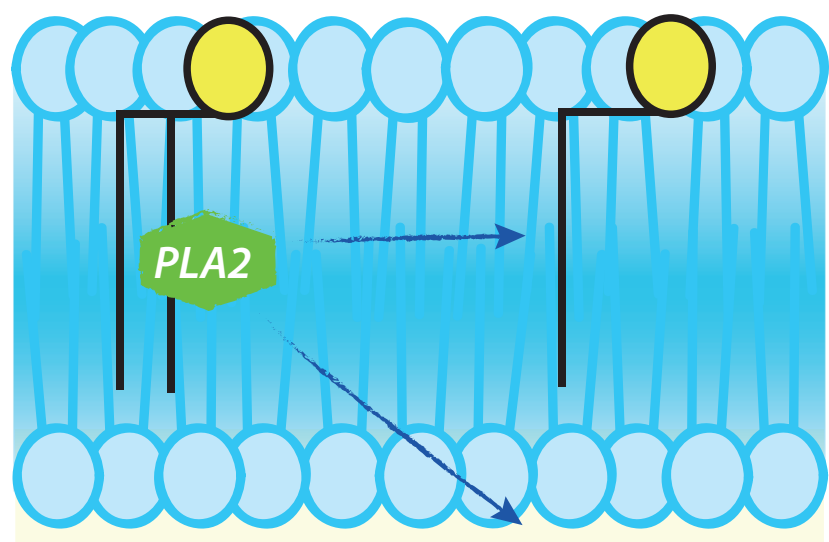

AA

Figure 3 
Figure 4

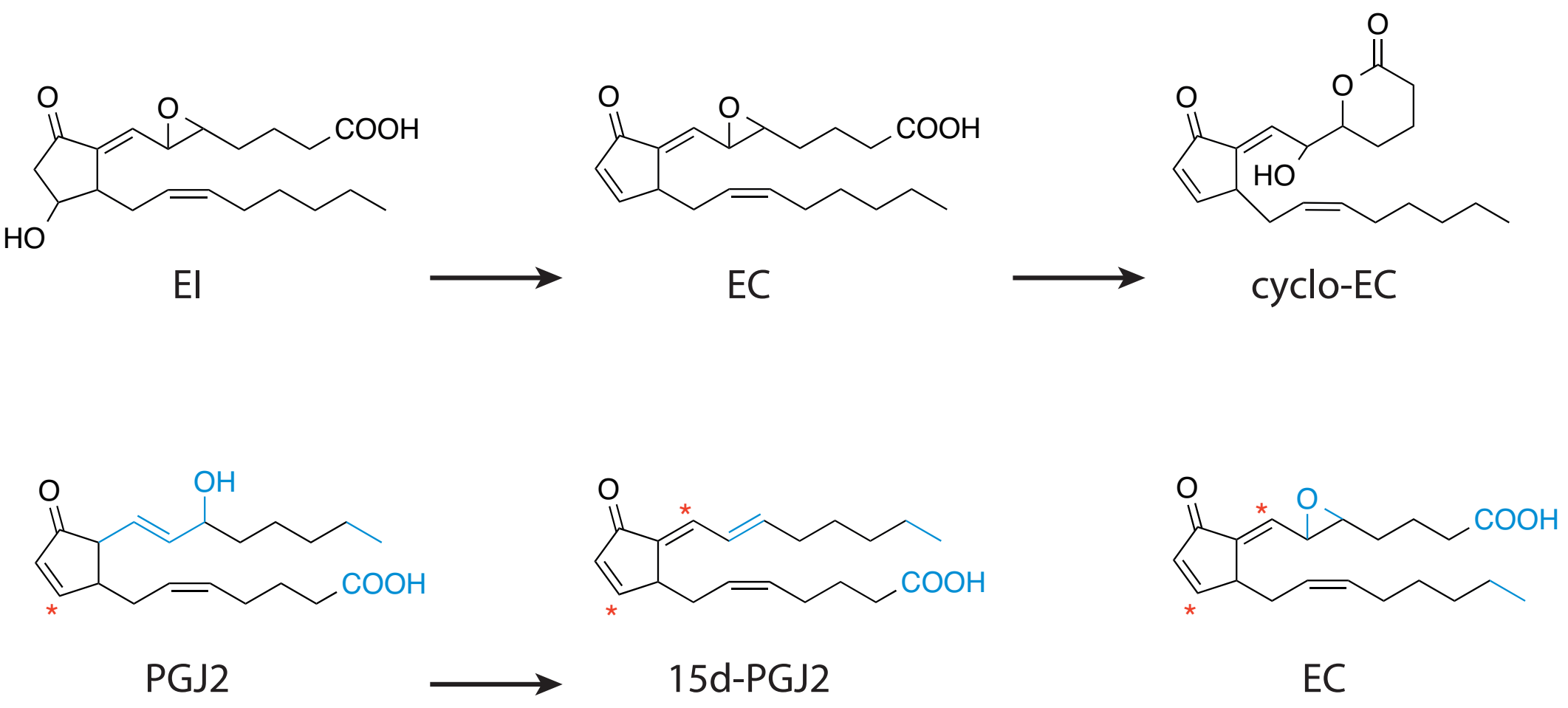

